\title{
HOW CAN BONE TUNNEL ENLARGEMENT IN ANTERIOR CRUCIATE LIGAMENT RECONSTRUCTION SURGERY BE MEASURED? DESCRIPTION OF A TECHNIQUE
}

Adriano Barros de Aguiar Leonardi ${ }^{1}$, Nilson Roberto Severino ${ }^{2}$, Aires Duarte Junior ${ }^{3}$

\begin{abstract}
Objective: To assess the presence of tibial bone tunnel enlargement after surgery to reconstruct the anterior cruciate ligament using quadruple flexor tendon grafts, and to propose a new technique for its measurement. Methods: The study involved 25 patients aged $18-43$ years over a sixmonth period. The assessment was based on radiographs taken immediately postoperatively and in the third and sixth months of evolution after operations to reconstruct the anterior cruciate ligament using grafts from the tendons of the semitendinosus and gracilis muscles, fixed in the femur with a transverse metal screw and in the tibia with an interference screw. The radiographs were evaluated in
\end{abstract}

terms of the relative value between the diameter of the tunnel and the bone, both at $2 \mathrm{~cm}$ below the medial tibial condyle. Results: There were significant increases in tunnel diameters: $20.56 \%$ for radiographs in anteroposterior view and $26.48 \%$ in lateral view. Enlargement was present in $48 \%$ of anteroposterior and lateral radiographs, but was present in both views in only $16 \%$ of the cases. Conclusions: Bone tunnel enlargement is a phenomenon found in the first months after surgery to reconstruct the anterior cruciate ligament. The measurement technique proposed in this study was sufficient to detect it.

Keywords - Anterior Cruciate Ligament/surgery; Tibia; Tendons; Femur; Muscles, Reconstructive Surgical Procedures

\section{INTRODUCTION}

Bone tunnel enlargement after surgery to reconstruct the anterior cruciate ligament has been a well documented phenomenon in the literature since the beginning of the 1990s. It comprises widening of the tibial and femoral tunnels on radiographs and other imaging examinations produced sequentially after the operation $^{(1)}$. Its incidence is extremely variable, ranging from $0 \%$ to $74.26 \%{ }^{(2)}$, and it is closely related to factors like the grafting, fixation and measurement methods used. For knees operated using hamstring tendons, the enlargement rates range from $11 \%$ to $73.9 \%$, compared with $2.1 \%$ to $47 \%{ }^{(3)}$ for knees operated using the patellar tendon. Taking into consideration the distance from the fixation to the joint surface, the rates range from $0 \%$ to $23 \%$ for grafts subjected to anatomical fixation ${ }^{(4)}$ and from $47 \%$ to $73.9 \%$ for those fixed at a distance from the joint surface ${ }^{(3)}$.

Although many studies have reported occurrences of enlargement, none have proven that it is clinically significant or that it is related to surgical failure rates ${ }^{(1,4,5,6)}$. Its mechanism is also not fully understood.

1 - MSc on Orthopedics and Traumatology from the School of Medical Sciences, Santa Casa de São Paulo. Trainee in the Sports Traumatology Group, Santa Casa de São Paulo, São Paulo, Brazil.

2 - PhD. Assistant Professor and Head of the Knee Group, Department of Orthopedics and Traumatology, School of Medical Sciences, Santa Casa de São Paulo (DOTFCMSCSP), São Paulo, Brazil.

3 - Head of the Sports Traumatology Group, Department of Orthopedics and Traumatology, School of Medical Sciences, Santa Casa de São Paulo (DOT-FCMSCSP), São Paulo, Brazil.

Work performed in the Department of Orthopedics and Traumatology, School of Medical Sciences, Santa Casa de São Paulo, "Fernandinho Simonsen" Wing (DOT-FCMSCSP). Director: Prof. Dr. Osmar Avanzi.

Correspondence: Avenida Eusébio Matoso, 196, Pinheiros - 05423-000 - São Paulo, SP. E-mail: leonardi@taktos.com.br

Trabalho recebido para publicação: July 09, 2010; aceito para publicação: December 23, 2010.

The authors declare that there was no conflict of interest in conducting this work 
Among the possible causes are mechanical factors such as mobility of the graft in the tunnel, localized stress at the tunnel entrance, incorrect tunnel positioning and aggressive rehabilitation ${ }^{(1,2,7)}$. The possible biological factors include nonspecific inflammatory responses mediated by cytokines, cell necrosis due to toxic products (ethylene oxide or metal), immune responses to foreign bodies (autologous grafts) and cell necrosis as a response to bone drilling using a bit ${ }^{(3)}$.

Statistically, there is greater incidence in tibial tunnels ${ }^{(8)}$. Although neither short-term nor long-term studies have correlated occurrences of enlargement with surgical failure $^{(9)}$, there is such concern in cases in which surgical revision might be necessary.

It seems that there is no consensus regarding the method for measuring enlargement on radiographs. While some authors have suggested that the tunnels should be measured at several points along their path $^{(7)}$, others have advocated that to diagnose and characterize bone tunnel enlargement, the distance between the two sclerotic margins should be measured in the region of their greatest dimension, at a standardized distance between the knee and the film holder, so as to avoid radiographic magnification $^{(10)}$.

The objectives of the present study were to assess the presence of tibial bone tunnel enlargement after surgery to reconstruct the anterior cruciate ligament and to describe an easily reproducible technique.

\section{SAMPLE AND METHODS}

\section{Study sample}

In a prospective study, we followed up 30 athletes who underwent anterior cruciate ligament reconstruction surgery by means of the video-arthroscopic technique using a quadruple graft from the gracilis and semitendinosus tendons, which were fixed by means of TransFix ${ }^{\circledR}$ transverse metal screws in the femur and interference screws in the tibia. In the final group, there were 23 male patients $(92 \%)$ and two female patients $(8 \%)$. The patients' mean age was 28.5 years, ranging from 18 to 43 years. The right side was operated in 16 patients $(64 \%)$ and the left side in nine patients $(36 \%)$, and the mean time that elapsed from the injury occurrence to the operation was 9.2 months, ranging from three to 25 months. The study was conducted between June 2006 and August 2008.

The patient inclusion criteria for the study were as follows:

a) Completely torn anterior cruciate ligament, diagnosed clinically and by means of magnetic resonance imaging (MRI);

b) Complaints of instability;

c) Other knee ligaments unaffected;

d) Joint cartilage unaffected;

e) Absence of injury to the posterior cornu of the menisci;

f) Absence of neurological and vascular lesions or previous fractures in the lower limbs;

g) Absence of unilateral load axis abnormalities;

h) No previous lesions to the locomotor system that were manifested as functional limitation, joint range of motion limitation or muscle functional abnormalities;

i) No previous surgery on the knee that was now to be operated.

\section{Postoperative follow-up}

For data gathering, we standardized the following observation times:

a. T0 - before the operation.

b. T1 - 0 to 30 days after the operation.

c. T2 - 3 months after the operation.

d. T3 - 6 months after the operation.

Radiographs were produced on the operated knees of each patient before the operation, in anteroposterior (AP) and lateral (L) views (Figures 1 and 2). The first postoperative radiographs were produced immediately after the operation (T1), soon after skin closure and application of the dressing. The others were produced three months (T2) and six months (T3) after the operation. The tunnel diameter was measured in the tibia $2.0 \mathrm{~cm}$ below the joint line in the medial tibial condyle, from the sclerotic margins of the visible path of the drill bit. By tracing out a line perpendicular to the tunnel, the variable "a" was generated. The values obtained were divided by the diameter of the bone, thus generating the constant "b", also measured $2.0 \mathrm{~cm}$ below the medial joint line. It was decided to generate relative results given by the ratio $\mathrm{a} / \mathrm{b}$ in order to avoid results biased by possible magnification of the radiograph.

The nonparametric Wilcoxon test was used to compare the variables found, as the ratio $a / b$ on the 
radiographs (AP1, AP2, AP3, L1, L2 and L3) in relation to the times (T0, T1, T2 and T3).

In all the tests, the significance level of $5 \%$ was used, such that tests with $\mathrm{p}<0.05$ were taken to be statistically significant. The results considered to be statistically significant were indicated by an asterisk $(*)$, and the non-significant results were labeled "ns".

\section{RESULTS}

Out of the 30 patients selected for the study, three were excluded because they did not attend the predetermined return visits, and another two were excluded because their imaging examinations were of poor quality, such that the tunnel diameters could not be measured.

By using the measurement method proposed here, and considering bone tunnel enlargement to be represented by increased bone tunnel diameter at successive measurements, i.e. T3 $>$ T2 $>$ T1, there were increases in bone tunnel diameter between all measurement times, except between $\mathrm{T} 2$ and $\mathrm{T} 3$ for radiographs in AP view (Table 1).

Although enlargement was present in $48 \%$ of the radiographs in AP and lateral views (Figure 1), we note on cross-correlating the data that enlargement became present in only $16 \%$ of the cases, i.e. in only nine patients. It was present in both radiographic views and was statistically significant for the time periods studied.

On expressing the phenomenon as percentages, we noted that there were increases of $20.56 \%$ on radiographs in AP view and $26.48 \%$ in lateral view from $\mathrm{T} 1$ to T3. In both views, we noted that the greatest intensity was over the first 12 weeks of the study period, and then it occurred less intensely until the sixth month (Figure 2).

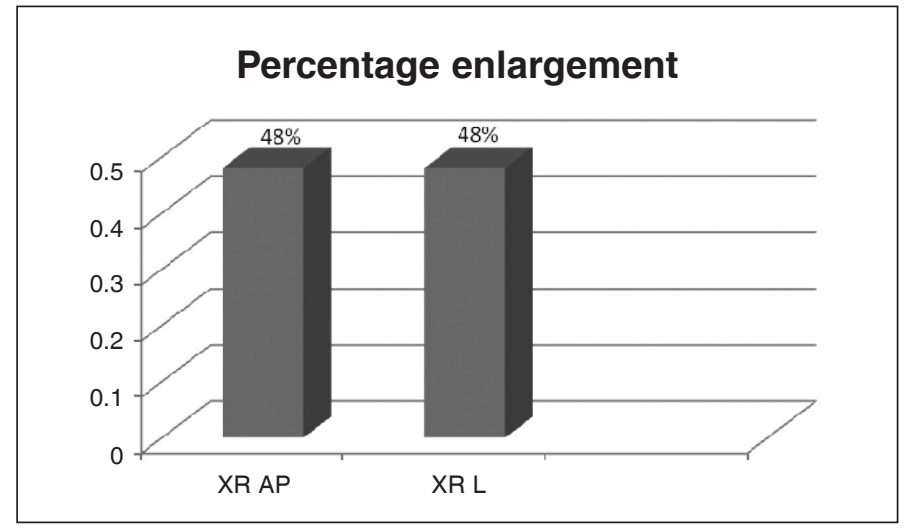

Figure 1 - Incidence of tibial bone tunnel enlargement on radiographs $(\mathrm{XR})$ in anteroposterior (AP) and lateral (L) views.

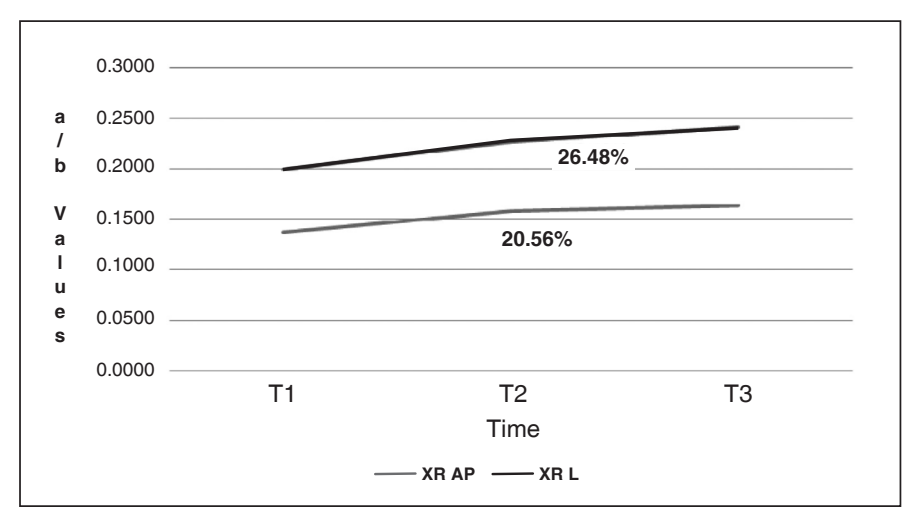

Figure 2 - Progression of bone tunnel enlargement, according to the $\mathrm{a} / \mathrm{b}$ relative values. $\mathrm{XR} \mathrm{AP}=$ radiograph in anteroposterior view; $X R L=$ radiograph in lateral view.

Wilcoxon test for AP view: Between T1 and T2: $p<0.001^{*}$ - Between T2 and T3: $p$ $=0.193 \mathrm{~ns}-$ Between T1 and T3: $p<0.001^{*}$

Wilcoxon test for lateral view: - Between T1 and T2: $p=0.06^{\star}$ - Between T2 and T3: $p=0.035^{\star}$ - Between T1 and T3: $p=0.004^{\star}$

\section{DISCUSSION}

Bone tunnel enlargement following surgery to reconstruct the anterior cruciate ligament is a phenomenon that has been reported over recent years, independent of the technique used ${ }^{(1,6,9)}$.

Its incidence has been described in different populations as ranging from $12.19 \%$ to $100 \%^{(1,11,12)}$.

Tabela 1 - Medidas resumo dos valores relativos entre o diâmetro do túnel ósseo e da largura da tíbia para as radiografias de incidência anteroposterior e perfil da tíbia em relação aos tempos T1 (pós-operatório imediato), T2 ( ${ }^{\circ}$ mês pós-operatório) e T3 (6 $6^{\circ}$ mês pós-operatório), em que RX AP significa radiografias de incidência anteroposterior e RXP, radiografias de incidência perfil.

\begin{tabular}{c|c|c|c|c|c|c|c|c|c|c}
\hline & \multicolumn{2}{|c|}{ Mean } & \multicolumn{2}{c|}{ Median } & \multicolumn{2}{c}{ SD } & \multicolumn{2}{c}{ Minimum } & \multicolumn{2}{c}{ Maximum } \\
\hline Time & XR AP & XR L & XR AP & XR L & XR AP & XR L & XR AP & XR L & XR AP & XR L \\
\hline T1 & 0.1368 & 0.1992 & 0.1411 & 0.1833 & 0.0175 & 0.0445 & 0.1105 & 0.1200 & 0.1625 & 0.3600 \\
\hline T2 & 0.1579 & 0.2263 & 0.1500 & 0.2222 & 0.0203 & 0.0312 & 0.1333 & 0.1791 & 0.2000 & 0.2830 \\
\hline T3 & 0.1630 & 0.2408 & 0.1625 & 0.2380 & 0.0235 & 0.0325 & 0.1200 & 0.1391 & 0.2000 & 0.2835 \\
\hline
\end{tabular}

Wilcoxon test for AP view: Between T1 and T2: $p<0.001^{*}$ - Between T2 and T3: $p=0.193$ ns - Between T1 and T3: $p<0.001^{*}$

Wilcoxon test for lateral view: Between T1 and T2: $p=0.06^{*}$ - Between T2 and T3: $p=0.035^{*}$ - Between T1 and T3: $\mathrm{p}=0.004^{*}$ 
We attribute this to the lack of consensus on how to measure it, the different methods used to obtain grafting material and fix it, the varying sizes of the samples studied and the individual criteria for defining bone tunnel enlargement.

In our study, we found the phenomenon in $48 \%$ of the radiographs in AP view and $48 \%$ of the radiographs in lateral view (Figure 1).

However, in cross-correlating the data, we noted that only $33.3 \%$ of the cases that presented enlargement in one radiographic view also showed it in the other view. In other words, only $16 \%$ of the patients presented enlargement that was present in both radiographic views studied. We attribute this to the diversity of format of the tunnels that underwent enlargement, which were classified as conical, concave or linear when analyzed three-dimensionally ${ }^{(1,13)}$.

In the literature, there is no consensus regarding the method to be used for measuring the enlargement. Some authors have stated that it should be measured at the sclerotic margins of the largest dimension of the bone tunnel, perpendicular to its long axis, on postoperative radiographs of the knee ${ }^{(7,11)}$. Others have favored always measuring a certain point below the joint interline ${ }^{(11)}$ or at different points along the tunnels because this better describes the morphology of the enlargement (conical, concave or linear) ${ }^{(13)}$. We also note that there is no consensus regarding how to express the results. While some authors do this in absolute numbers ${ }^{(14)}$, others express the enlargement as percentages ${ }^{(3,7,12)}$.

In a comparative study in which different observers analyzed the same radiographs to determine the bone tunnel enlargement, each using the parameter that they thought was best, Webster et $\mathrm{al}^{(14)}$ found that the results varied from $16 \%$ to $24 \%$.

We chose to use the method of Fahey and Indelicato $^{(5)}$ and standardized on a point $2 \mathrm{~cm}$ below the medial joint interline, the location where the phenomenon seems to occur with greatest intensity ${ }^{(1,12)}$. All the images were analyzed by the same examiner.

To deal with the radiographic magnification effect, in which the image obtained on the film becomes bigger than the object that is being studied, some authors have recommended that a correction factor should be calculated, and have estimated that for conventional radiographs, the value calculated should be up to $10 \%{ }^{(7,10)}$.
In our study, we created a method in which we obtained relative values between the tunnel diameter, which we called variable "a", and its long axis, which we called constant "b" (Figures 3 and 4), both measured $2 \mathrm{~cm}$ below the joint interline. We believe that this method not only prevents bone tunnel measurement errors caused by magnification on the radiographs, but also avoids errors caused by attempting to establish the point of greatest tunnel diameter, since we believe that the latter could lead to making measurements at different sites in the same knee, which would obviously lead to erroneous results.

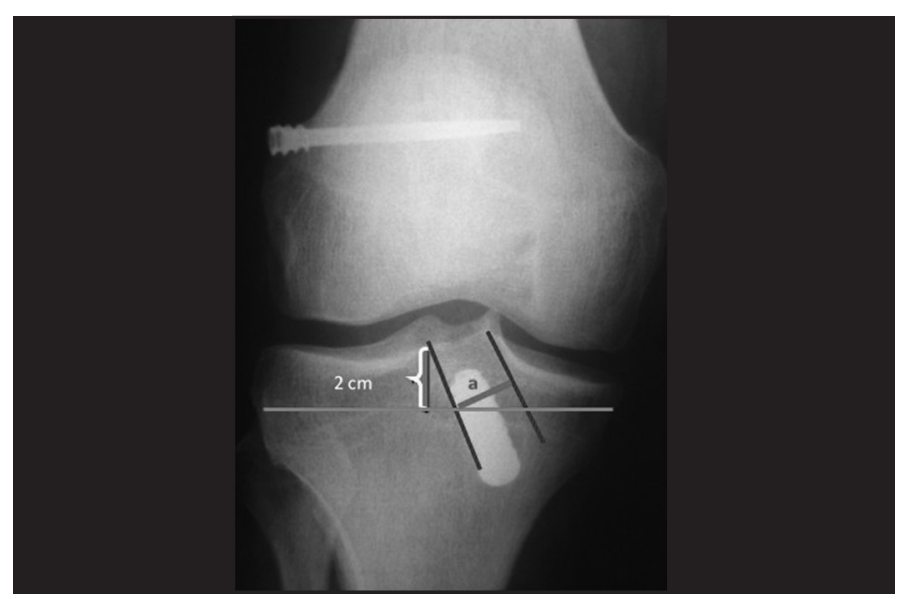

Figure 3 - Radiograph in anteroposterior (AP) view. The blue line follows along the margins of the tunnel, $2 \mathrm{~cm}$ below the medial joint line, and the tunnel diameter is given as the perpendicular to this blue line, represented by the red line "a". At the same point, the bone diameter is calculated, represented by the orange line "b". The variable "a" is then divided by the constant "b".

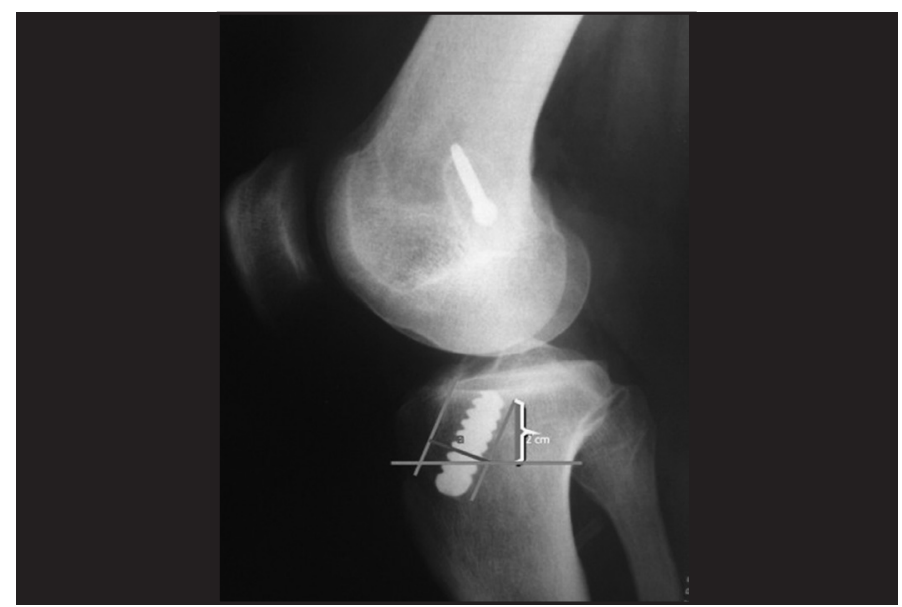

Figure 4 - Radiograph in lateral (L) view. The blue line follows along the margins of the tunnel, $2 \mathrm{~cm}$ below the medial joint line, and the tunnel diameter is given as the perpendicular to this blue line, represented by the red line "a". At the same point, the bone diameter is calculated, represented by the orange line "b". The variable "a" is then divided by the constant "b". 
Authors who have compared measurements on bone tunnel enlargement made using radiographs and computed tomography scans have unanimously affirmed that radiographs may underestimate the true diameter of tunnel enlargement, especially during the first three months, because of the technical difficulty in determining the sclerotic margins of the tunnels, especially in femoral tunnels ${ }^{(11,13,14)}$.

In our study, we made the evaluations using radiography because this is the most popular, least expensive and most widely used imaging examination, and it is certainly the most accessible examination for carrying out similar studies. We believe that this continues to be an excellent method for evaluating enlargement because, unlike in the findings of some of these authors ${ }^{(11,13,14)}$, we noted the presence of the phenomenon within the first three months. It was statistically significant and occurred more rapidly than over the period from the third to the sixth month (Table 1 and Figure 1).

In relation to the time at which tunnel enlargement appears, there seems to be a consensus that it may occur within the first year after the surgery, especially between the third and ninth weeks, and that, two to three years after the operation, either it is no longer occurring or it is occurring only very slightly ${ }^{(4,9)}$. We made a six-month follow-up, which according to the literature is the ideal length of time for ascertaining whether the phenomenon is occurring. We noted that the enlargement was significant and more intense over the first three months, and that it was less intense from three to six months after the operation. The findings were statistically significant for lateral-view radiographs on the tibia but not significant for AP radiographs on the tibia (Table 1 and Figure 2).

\section{CONCLUSION}

Bone tunnel enlargement is a phenomenon found in the first months after surgery to reconstruct the anterior cruciate ligament. The measurement technique proposed in this study was sufficient to detect it.

\section{REFERENCES}

1. Höher J, Möller HD, Fu FH. Bone tunnel enlargement after anterior cruciate ligament reconstruction: fact or fiction? Knee Surg Sports Traumatol Arthrosc. 1998;6(4):231-40.

2. Buelow JU, Siebold R, Ellermann A. A prospective evaluation of tunnel enlargement in anterior cruciate ligament reconstruction with hamstrings: extracortical versus anatomical fixation. Knee Surg Sports Traumatol Arthrosc. 2002;10(2):80-5.

3. Clatworthy MG, Annear P, Bulow JU, Bartlett RJ. Tunnel widening in anterior cruciate ligament reconstruction: a prospective evaluation of hamstring and patella tendon grafts. Knee Surg Sports Traumatol Arthrosc. 1999;7(3):38-45.

4. Barber FA, Spruill B, Sheluga M. The effect of outlet fixation on tunnel widening. Arthroscopy. 2003;19(5):485-92.

5. Fahey M, Indelicato PA. Bone tunnel enlargement after anterior cruciate ligament replacement. Am J Sports Med. 1994;22(3):410-4.

6. Zijl JA, Kleipool AE, Willems WJ. Comparison of tibial tunnel enlargement after anterior cruciate ligament reconstruction using patellar tendon autograft or allograft. Am J Sports Med. 2000;28(4):547-51.

7. Zysk SP, Fraunberger P, Veihelmann A, Dörger M, Kalteis T, Maier M, et al. Tunnel enlargement and changes in synovial fluid cytokine profile following anterior cruciate ligament reconstruction with patellar tendon and hamstring tendon autografts. Knee Surg Sports Traumatol Arthrosc.2004;12(2):98-103.

8. L'Insalata JC, Klatt B, Fu FH, Harner CD. Tunnel expansion following anterior cruciate ligament reconstruction: a comparison of hamstring and patellar tendon autografts. Knee Surg Sports Traumatol Arthrosc. 1997;5(4):234-8.

9. Berg EE, Pollard ME, Kang Q. Interarticular bone tunnel healing. Arthroscopy. 2001;17(2):189-95.

10. Camanho GL, Camanho LF, Aguiar AFM. Alargamento dos túneis ósseos nas reconstruções do ligamento cruzado anterior com tendões flexores: avaliação de 34 pacientes com quatro anos de seguimento. Rev Bras Ortop. 2004;39(5):214-22.

11. Fink C, Zapp M, Benedetto KP, Hackl W, Hoser C, Rieger M. Tibial tunnel enlargement following anterior cruciate ligament reconstruction with patellar tendon autograft. Arthroscopy. 2001;17(2):138-43.

12. Rodeo SA, Kawamura S, Kim HJ, Dynybil C, Ying L. Tendon healing in a bone tunnel differs at the tunnel entrance versus the tunnel exit: an effect of grafttunnel motion? Am J Sports Med. 2006;34(11):1790-800

13. Granata Júnior G, Caraviello A, Granata GSM, Nakano E, Matoso F, Navarro R, et al. Enlargement of bone tunnels in the anterior cruciate ligament reconstruction with semitendinosus and gracilis: compare tridimensional computadorized tomogaphy and radiography. (Poster P15-1066] Knee Surg Sports Traumatol Arthrosc. 2008; 16(Suppl.1):S80-S230. [Abstracts of the 13th ESSKA 2000 Congress, May 21-24, 2008, Porto, Portugal).

14. Webster KE, Feller JA, Elliott J, Hutchison A, Payne R. A comparison of bone tunnel measurements made using computed tomography and digital plain radiography after anterior cruciate ligament reconstruction. Arthroscopy. 2004;20(9):946-50. 